\title{
Quality of Systematic Reviews of the Foods with Function Claims in Japan: Comparative Before- and After-Evaluation of Verification Reports by the Consumer Affairs Agency
}

\author{
Hiroharu Kamioka ${ }^{1, *}$, Kiichiro Tsutani ${ }^{2}$, Hideki Origasa ${ }^{3}$, Takahiro Yoshizaki ${ }^{4}$, \\ Jun Kitayuguchi ${ }^{5}$, Mikiko Shimada ${ }^{6}$, Yasuyo Wada ${ }^{7}$ and Hiromi Takano-Ohmuro ${ }^{8}$ \\ 1 Faculty of Regional Environment Science, Tokyo University of Agriculture, 1-1-1 Sakuragaoka, Setagaya-ku, \\ Tokyo 156-8502, Japan \\ 2 Tokyo Ariake Medical and Health Sciences University, 2-9-1 Ariake, Kouto-ku, Tokyo 135-0063, Japan \\ 3 Division of Biostatistics and Clinical Epidemiology, University of Toyama School of Medicine, 2630 Sugiya, \\ Toyama City, Toyama 930-0194, Japan \\ 4 Faculty of Food and Nutritional Sciences, Toyo University, 1-1-1 Izumino, Itakura Town, Gunma 374-0193, \\ Japan \\ 5 Physical Education and Medicine Research Center Unnan, 328 Uji, Unnan City, Shimane 699-1105, Japan \\ 6 Department of Nutrition, Faculty of Health Care, Kiryu University, 606-7 Asami, Midori City, \\ Gunma 379-2329, Japan \\ 7 Department of Food and Nutrition, Faculty of Human Life, Jumonji University, 2-1-28 Sugasawa, Niiza City, \\ Saitama 352-8510, Japan \\ 8 Research Institute of Pharmaceutical Sciences, Musashino University, 1-1-20 Aramachi, Nishitokyo City, \\ Tokyo 202-8585, Japan \\ * Correspondence: h1kamiok@nodai.ac.jp
}

Received: 27 May 2019; Accepted: 10 July 2019; Published: 12 July 2019

\begin{abstract}
Background: In Japan, a new type of foods with health claims, called Foods with Function Claims (FFC), was introduced in April 2015 in order to make more products available that are clearly labeled with certain health functions. Regarding substantiating product effectiveness, scientific evidence for the proposed function claims must be explained by systematic reviews (SRs), but the quality of SRs was not clear. The objectives of this review were to assess the quality of SRs based on the FFC registered on the Consumer Affairs Agency (CAA) website in Japan, and to determine whether the CAA's verification report in 2016 was associated with improvement in the quality of SRs. Methods: We evaluated the reporting quality of each SR by the AMSTAR checklist on methodological quality. We searched the database from 1 April to 31 October 2015 as the before-SR and from 1 July 2017 to 31 January 2018 as the after-SR. Results: Among the 104 SRs reviewed, 96 final products were included: $51(53.1 \%)$ were supplements, $42(43.8 \%)$ were processed foods without supplements, and $3(3.1 \%)$ were fresh foods. Of the 104 SRs, $92(88.5 \%)$ were qualitative reviews (i.e., without meta-analysis) and $12(11.5 \%)$ performed a meta-analysis. The average quality score of before-SRs and after-SRs was $6.2 \pm 1.8$ and $5.0 \pm 1.9$, respectively, a statistically significant decrease $(p<0.001)$. Conclusion: Overall, the methodology and reporting quality of after-SRs based on the FFC were poorer than those of before-SRs. In particular, there were very poor descriptions and/or implementations of study selection and data extraction, search strategy, evaluation methods for risk of bias, assessment of publication bias, and formulating conclusions based on methodological rigor and scientific quality of the included studies.
\end{abstract}

Keywords: quality; systematic review; health claim; supplement 


\section{Background}

The Codex Alimentarius Commission (CAC) is an intergovernmental organization that was founded in 1962 to develop food standards, guidelines, and codes of practice [1]. The basic principles of CAC are that health claims should be substantiated by currently sound and sufficient scientific evidence, provide truthful and nonmisleading information that consumers can use to choose healthy diets, and be supported by specific consumer education [2].

In accordance with CAC guidelines, only government-approved Foods for Specified Health Uses (FOSHU) and foods with nutrient function claims (FNFC) can make function claims on food labels in Japan, and these must comply with specifications and standards designated by the government [3]. The FOSHU are scientifically accepted for their usefulness in maintaining and promoting health and are therefore permitted to contain food effects and safety claims that have been evaluated by the government.

Foods approved as FNFC can be used to supplement or complement the nutrients (vitamins, minerals, etc.) that are in insufficient quantities in an individual's daily diet. These foods can carry a nutrient function claim prescribed by the government standards and can be freely manufactured and distributed without any permission from or a notification to the national government [3]. In addition to these categories, a new type of foods with health claims, called Foods with Function Claims (FFC), was introduced in April 2015 (Figure 1). The FFC allows manufacturers to submit labeling to the Secretary-General of the Consumer Affairs Agency (CAA) in Japan that indicates the food is expected to have a specific effect on health, except for reducing the risk of diseases.

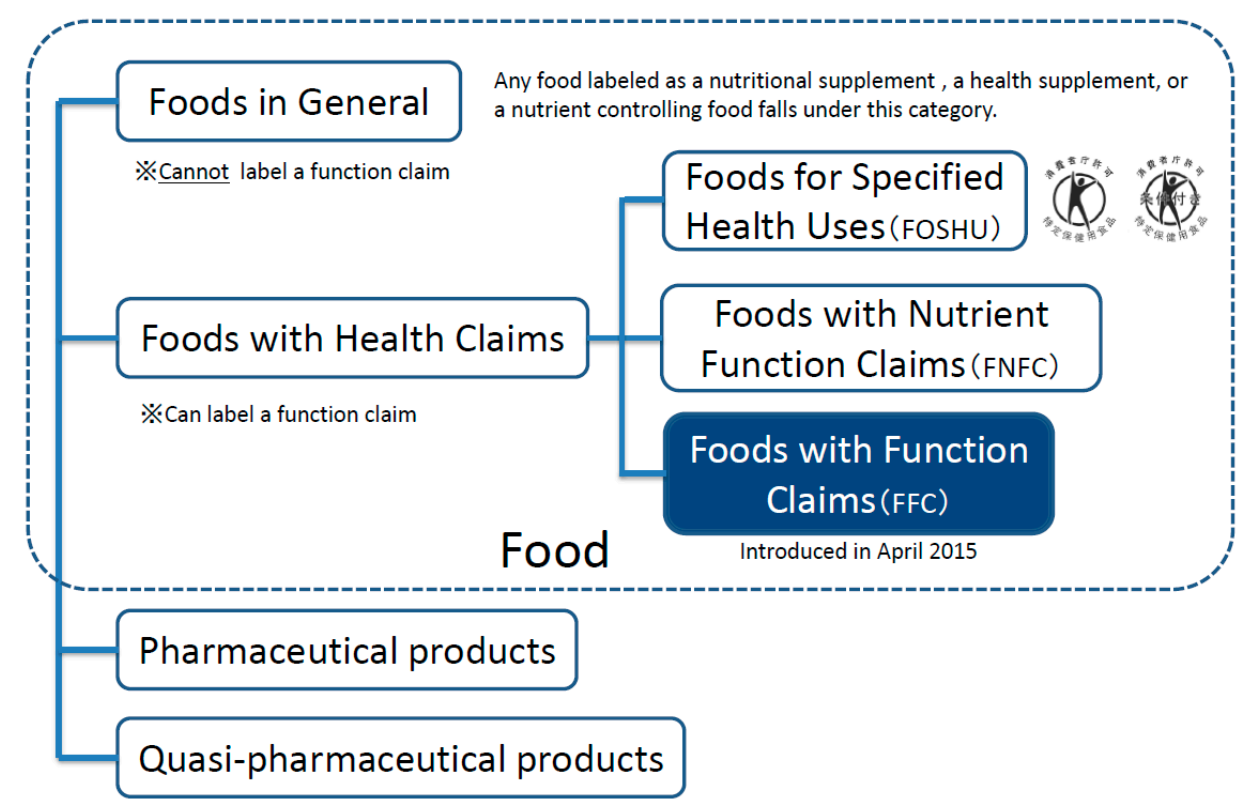

Figure 1. Food labeled with certain nutritional or health functions in Japan.

Unlike the strict evaluation criteria applied through the FOSHU and FNFC processes, the FFC is only a notification system in which food manufacturers must meet five unique and specific criteria (Table 1). Although the government does not evaluate the safety and effectiveness of the submitted product, i.e., it does not utilize a notification system, the industry (applicant) must fulfill several procedures to submit a notification. All the FFC criteria submitted by the manufacturers are disclosed on the website of the CAA, which gives approval for the labeling of food products. For a food product to claim effectiveness on its label, evidence for its proposed function claims must be substantiated by one of two standard scientific methods: clinical trials such as randomized controlled trials (RCTs), or systematic reviews (SRs). Details about the use of these two methods for food with function claims have been published on the CAA website [4]. A notable point in this system is that not only RCTs but 
also SRs are permitted. Since promoting deregulation is a national goal, SRs have the advantage of being easy to report to small and medium-sized enterprises because they are less expensive than RCTs.

Table 1. Characteristics of the foods with function claims in Japan.

\begin{tabular}{cl}
\hline 1 & $\begin{array}{l}\text { Foods with Function Claims are for people not suffering from any disease (excluding minors, } \\
\text { pregnant women (and those planning a pregnancy), or lactating women). }\end{array}$ \\
\hline 2 & All food products including fresh produce are subject to this system. ${ }^{*}$ \\
\hline 3 & $\begin{array}{l}\text { Prior to market entry (before at least } 60 \text { days), food business operators are required to submit } \\
\text { information, such as on food safety and effectiveness and the system in place to collect information } \\
\text { on adverse health effects, to the Secretary-General of Consumer Affairs Agency. }\end{array}$ \\
\hline $4 \quad \begin{array}{l}\text { Unlike Foods for Specified Health Uses, the government does not evaluate the safety and } \\
\text { effectiveness of the submitted product, i.e. notification system. }\end{array}$ \\
\hline $5 \quad$ The submitted all information is disclosed on the website of the Consumer Affairs Agency. ${ }^{* *}$ \\
\hline $\begin{array}{l}\text { Modified partially for this study based on the Consumer Affairs Agency website in Japan. * Excluding Foods for } \\
\text { Special Dietary Uses (including FOSHU), FNFC, alcohol-containing beverages, and food products that may lead to } \\
\text { the excessive consumption of fat, cholesterol, sugar (limited to mono- and disaccharides, excluding sugar alcohols), } \\
\text { or sodium. }{ }^{* *} \text { The all information was only written in Japanese. }\end{array}$
\end{tabular}

The methodology of an SR with or without meta-analysis may not be familiar to general or nutritional researchers in the food industry. An SR addresses a question that is carefully formulated to be answered by analysis of all available evidence. It performs an objective literature search by applying predetermined inclusion and exclusion criteria to critically appraise what literature is relevant [5]. The SR is an important method that can help researchers to identify evidence of an effective intervention from a large volume of published biomedical literature.

However, although the methodology of an SR is important in terms of evidence-based nutrition (EBN), an SR has the weakness that assessment of fresh foods that most people eat daily is very difficult. Additionally, an SR may be of limited use if the methods used to conduct the SR are flawed and reporting of the SR was incomplete [6]. Moreover, the scientific validity of an SR is based on deductive planning and clear documentation of the methodological approach that was employed to design and conduct the SR [7]. We were interested in evaluating whether or not SRs of the FFC, which is based on a notification system, had been conducted by appropriate scientific methods, and we hoped to formulate a research challenge for future SRs of the FFC.

In our previous study [8], we adopted a well-known measurement tool for the 'assessment of multiple SR' (AMSTAR checklist) [9] and assessed the quality of 49 SRs that were based on the FFC registered on the CAA website from 1 April to 27 October 2015. Results from that study showed that the methodology and reporting quality of SRs were in the poor description category (mean \pm SD: $6.2 \pm 1.8$ points, range $2-11$ points for 11 points full-mark). Based on scientific quality, the SRs had very poor descriptions and/or implementation of the registration, poor evaluations of publication bias, and questionable conclusions.

On the other hand, the CAA in 2016 formatted the expert working group (methodologists for SR) in order to extract issues for appropriate operation of the FFC system and perform a verification [10] according to the PRISMA [11]. Fifty-one submitted SRs were selected for evaluation of quality. These SRs were all registered on the CAA website from 1 April to 31 October 2015. To complete basic standard-level SRs, considering the difficulty in handling foods, this project team attached "appropriate description in SRs based on the 'PRISMA Checklist: an extended version for submitted SRs of Foods with Function Claims'" to its final report and included detailed proposals and examples. Most authors of the present study (HK, HO, TK, JK, MS, and HTO) also participated in this CAA project. Since the report was a specific guideline to perform and submit new SRs and was submitted to food business operators in Japan, we assumed that all researchers in this field watched it closely, followed the checklist, and completed an appropriate description afterward. Therefore, we hypothesized that the CAA's 2016 verification report based on PRISMA [10], in addition to our article on quality evaluation in 2017 [8], were associated with improvement in the quality of subsequently submitted SRs. 
The present study design was based on a previous comparative before- and after -evaluation in which RCTs published in 1994 (pre-CONSORT) were compared with RCTs published in the same journals in 1998 (post-CONSORT) [12].

The objectives of this review were to assess the quality of SRs based on the FFC registered on the CAA website in Japan, and to determine whether the CAA's verification report in 2016 was associated with improvement in the quality of SRs.

\section{Methods}

\subsection{Scope of This Review}

The basic scientific approach of the FFC system ensures safety, functionality, and effectiveness. The purpose of this study was to assess only the quality of SRs, and it therefore focused on face and contact validity for measuring the methodological quality of SRs. Whether each product or functional substance involved is effective is a separate research issue and was not in the scope of this study.

The PRISMA statement is a respected reporting guideline designed to improve the completeness of an SR report [11]. Furthermore, although there was a new critical appraisal tool for SRs (AMSTAR 2) [13], we already performed the before-evaluation based on the AMSTAR checklist [9]. Therefore, we performed the after-evaluation with the same tool in order to compare the evaluation results.

\subsection{Criteria for Considering Studies Included in This Review}

Criteria for considering studies that were included in this review were based on those in the predefined protocol.

\subsection{Types of Studies}

Studies were eligible if they were SRs (with or without a meta-analysis).

\subsection{Types of Participants}

This study was a review based on SRs and was therefore restricted to original SRs of healthy adults (people not suffering from any disease).

\subsection{Comparator(s)/Control}

In the original SRs, controls were defined as healthy adults identified from preplanned stratified analyses of (a) placebo controls or waiting list controls, (b) intervention groups that compared different types of products or ingredients, and (c) low- or medium-level intake groups of the same product or ingredient.

\subsection{Types of Intervention and Language}

For processed foods in the form of supplements, studies included at least one intervention group in which the functional ingredient and the final product were applied. For fresh food and other processed food, studies included at least one exposure group in which the functional ingredient and the final product were applied and included observational studies and intervention studies.

\subsection{Types of Outcome Measures}

Outcome measures included many types of positive contributions to health, to the improvement of a function, or to preserving health as an outcome. In effect, we included all notified SRs.

\subsection{Search Methodology for Identification of Studies}

\subsubsection{Search Strategies}

Our search of the databases on the CAA website covered the period from 1 April 2015 (starting date) through 27 October 2015 for the before-SRs [8], and from 1 July 2017 to 31 January 2018 for the 
after-SRs. The special search strategies contained the elements and terms (i.e., a specific search method based on keywords) on the CAA website. All references in identified SRs were screened. The search was performed by the steering author (HK).

\subsubsection{Hand-Searching and Reference Checking}

Since this study was limited to SRs registered in the CAA database, hand-searching and reference checking were not applicable.

\subsection{Review Methods}

\subsubsection{Selection of Studies}

To select the studies that were to be reviewed, all criteria were applied by the steering author (HK) to the full library of articles published on the CAA website. Studies were selected when (i) the design was an SR based on an intervention study, (ii) the study was appropriately notified by the CAA, and (iii) the study was published on the website. Studies (notification) that were excluded are presented with reasons for exclusion.

\subsubsection{Quality Assessment of Included Studies}

To ensure that variation was not caused by systematic errors in study design or execution, four review authors (YW, TY, MS, and JK) independently assessed the quality of articles (i.e., every two reviewers were paired). Disagreements and uncertainties were resolved by discussion with another author (HK). A full quality appraisal of these papers was made using a combined tool based on the AMSTAR checklist that was developed to assess methodological quality of SRs. Each item was scored as 'present' (Yes), 'absent' (No), 'unclear or inadequately described' (Can't answer), or 'Not applicable' (n/a). Depending on the study design (with or without meta-analysis), some items were not applicable; therefore, the "n/a" score was not considered to be an error in the calculation for quality assessment. Although the original AMSTAR has 11 check items, two meanings can be applied to item \#3, which reads as follows: "Was a comprehensive literature search performed. At least two electronic sources should be searched. The report must include years and databases used. Key word and/or MESH terms must be stated and where feasible the search strategy should be provided (continued)." Because the guideline for FFC notification on the CAA website requires the use of at least two electronic databases, we divided \#3 into two parts in order to detect any trend arising from the use of databases: "\#3a; which databases did the SR use or number of the other databases", and "\#3b; Did the SR use MESH terms and related search function to detect comprehensively".

All authors attended one 3-hour consensus-training session based on the AMSTAR checklist before starting the quality assessment to ensure that they used the same criteria and correctly evaluated the check-items for an SR.

The percentage of descriptions present on all 11 (excluding \#3a) of the check items for the quality assessment of articles was determined. Then, based on the percentage of risk of poor methodology and/or bias, each item was assigned to one of the following categories: good description $(80 \%-100 \%)$, poor description (50-79\%), or very poor description (0-49\%).

Disagreements and uncertainties were resolved by discussion with other authors (HK, TK, and $\mathrm{HO}$ ). Interrater reliability was calculated by the steering author (HK) on a dichotomous scale using percentage agreement and Cohen's kappa coefficient $(k)$.

\subsubsection{Characteristics of Studies and Data Extraction}

Two authors (HK and $\mathrm{HO}$ ) described the characteristics from each article based on information on the CAA website but did not produce a structured abstract for SRs, which is recommended [14]. Because this study focused on evaluating quality of SRs of the FFC, it did not summarize evidence for the effectiveness of each SR. 


\subsubsection{Research Protocol Registration}

We submitted and registered our research protocol to PROSPERO (CRD42017080833) and UMIN-CTR (UMIN000029821). PROSPERO is an international database of prospectively registered SRs in health and social care [15]. Key features from a review protocol are recorded and maintained as a permanent record in PROSPERO. UMIN-CTR is a Japanese and international database of prospectively registered clinical trials and other trials with SRs in health and social care and was accepted as an international registry database by the International Committee of Medical Journal Editors in 2007. In a previous study [8], we implemented our protocol before UMIN-CTR was formally launched on 1 April 2015, and we planned to continue checking target SRs prospectively from our study start date. In the present study, we also planned to continue reviewing target SRs prospectively from 1 July 2017 to 31 January 2018.

\subsubsection{Statistical Analysis}

A two-sample $t$ test was employed for comparisons between two terms (number of databases and before- and after-evaluation scores) with continuous variables in the analysis. The $\chi^{2}$ test and Fisher's exact test were performed with discrete variables (i.e., number and $\%$ of good description on each item). Statistical analysis was performed with SPSS version 23.0 (IBM Corporation, Armonk, NY, USA) for Windows. For all analyses, $p$-values less than 0.05 were considered statistically significant.

\section{Results}

\subsection{Study Selection and Characteristics}

Of the 294 potentially relevant articles included in the literature search, 198 notifications were excluded because they did not meet the eligibility criteria (Figure 2). A total of 104 SRs (including eight multiple claims) met all inclusion criteria. The language of all eligible publications was Japanese.

The contents of all articles were summarized as brief characteristics (Table 2). Among the 104 SRs reviewed, 96 final products were included: 51 (53.1\%) were supplements, $42(43.8 \%)$ were processed foods without supplements, and $3(3.1 \%)$ were fresh foods. Of the 104 SRs, 91 (87.5\%) were qualitative reviews (i.e., without meta-analysis), and 13 (12.5\%) performed a meta-analysis.

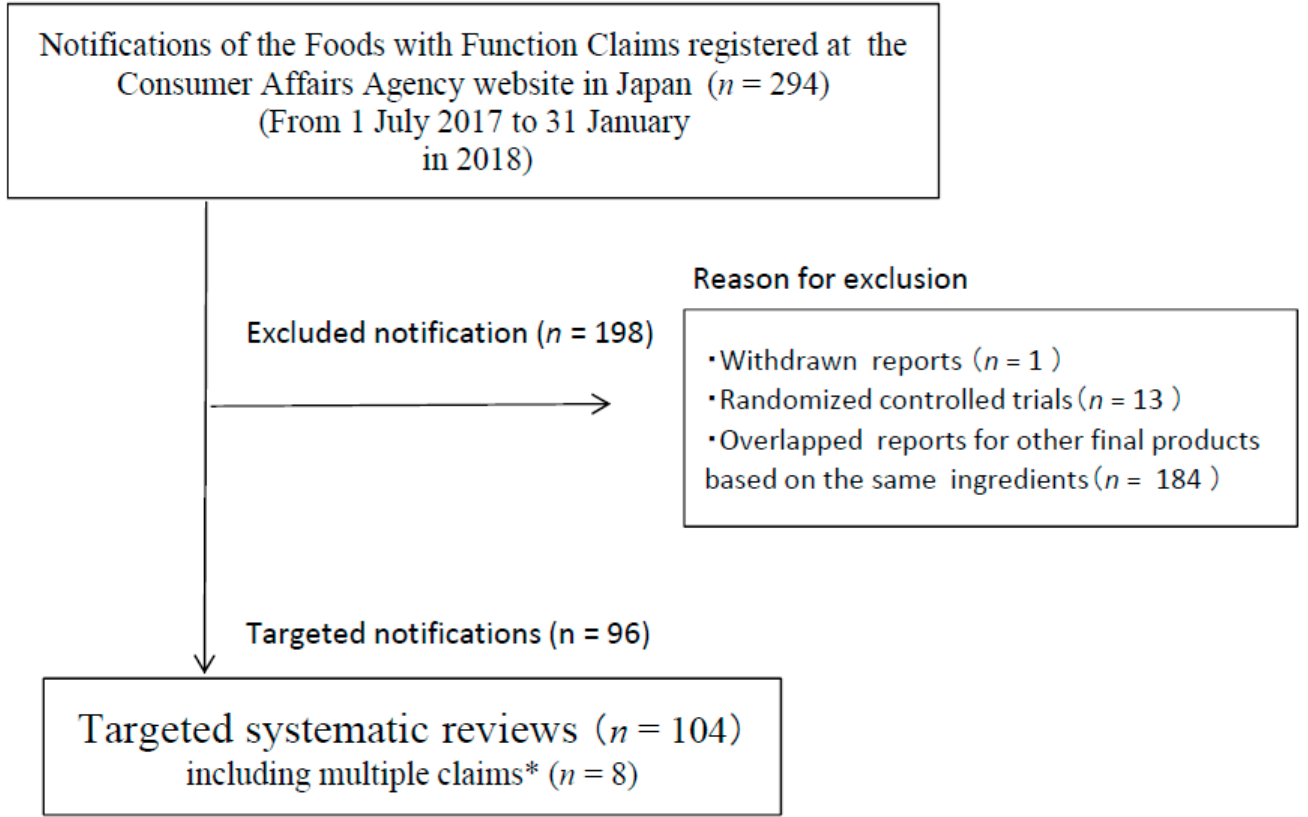

Figure 2. Flowchart of trial process. * A product has more than one functionality (e.g., decreased body fat and increased HDL cholesterol) and is displaying them. 
Table 2. Characteristics of systematic reviews of the foods with function claims.

\begin{tabular}{|c|c|c|c|c|}
\hline No. * & Product Name & $\begin{array}{l}\text { Food Business } \\
\text { Operator }\end{array}$ & $\begin{array}{c}\text { Classification of Food } \\
1 \text { Supplement } \\
2 \text { Processed Food } \\
3 \text { Fresh Produce }\end{array}$ & Functional Substance \\
\hline C48 & Ayumu Chikara (Fruit Yogurt) & Lion Corporation & 2 & $\begin{array}{l}\text { HMB( } \beta \text {-hydroxy- } \beta \text {-methylbutyrate), } \\
\text { Glucosamine hydrochloride }\end{array}$ \\
\hline C49 & Kangengata CoQ10 150 & FINE JAPAN Co., Ltd. & 1 & Reduced coenzyme Q10 \\
\hline C50 & DESK RAKU & Nihon kefir Co., Ltd. & 1 & Lutein \\
\hline C51 & OFURO TIME cocktail taste salty dog taste & KING BREWING Co., Ltd. & 2 & Indigestible dextrin (dietary fiber) \\
\hline C52 & OFURO TIME cocktail taste cassis orange taste & KING BREWING Co., Ltd. & 2 & Monoglucosyl hesperidin \\
\hline C53 & Karada ni yasashii mizu (lemon taste) & Melodian Co., Ltd. & 2 & Hyaluronic acid $\mathrm{Na}$ \\
\hline C54 & Karada ni yasashii mizu (peach taste) & Melodian Co., Ltd. & 2 & Indigestible dextrin (dietary fiber) \\
\hline C55 & Karada ni yasashii mizu (grapefruit taste) & Melodian Co., Ltd. & 2 & Salacinol made from Salacia \\
\hline C59 & The product has not been named in English yet. & Kyoto Pharmaceutical Industries, Ltd. & 1 & $\begin{array}{c}\text { G biloba flavonoid glycoside, } \mathrm{G} \text { biloba } \\
\text { terpene latone }\end{array}$ \\
\hline $\mathrm{C} 60$ & DHA no kiwami 1000 mg plus & Bizen Chemical Co., Ltd. & 1 & DHA, EPA \\
\hline C61 & SUNKINOU Fish oil & Sunsho Pharmaceutical Co., Ltd. & 1 & DHA, EPA \\
\hline C62 & Kotsukotsu Soybean Isoflavone & KYOWA YAKUHIN Co., Ltd. & 1 & Soy isoflavone \\
\hline C63 & DHA no kiwami 1000 mg plus W & Bizen Chemical Co., Ltd. & 1 & DHA, EPA \\
\hline C65 & EGAO GABA Stress Care & EGAO Co., Ltd. & 1 & GABA ( $\gamma$-Aminobutyric acid $)$ \\
\hline C68 & SUNKINOU Bifidobacteria & Sunsho Pharmaceutical Co., Ltd. & 1 & Bifidobacterium longum BB536 \\
\hline C69 & Yumemin & SIMANOYA Co., Ltd. & 1 & L-Theanine \\
\hline $\mathrm{C} 70$ & meiji GABA COFFEE & Meiji Co., Ltd. & 2 & GABA \\
\hline C71 & Glucosamine2000 KAIHO & NIHON_YAKUSHIDO Co., Ltd. & 1 & Glucosamine hydrochloride \\
\hline $\mathrm{C} 72$ & Ginkgo Extract & Yuuki Medicine manufacture, Inc. & 1 & $\begin{array}{l}\text { G biloba flavonoid glycoside, } \mathrm{G} \text { biloba } \\
\text { terpene latone }\end{array}$ \\
\hline $\mathrm{C} 73$ & Soup with Brown rice Chinese soup & SHOKKYO Co., Ltd. & 2 & Indigestible dextrin (dietary fiber) \\
\hline
\end{tabular}


Table 2. Cont

\begin{tabular}{|c|c|c|c|c|}
\hline No. * & Product Name & $\begin{array}{l}\text { Food Business } \\
\text { Operator }\end{array}$ & $\begin{array}{l}\text { Classification of Food } \\
1 \text { Supplement } \\
2 \text { Processed Food } \\
3 \text { Fresh Produce }\end{array}$ & Functional Substance \\
\hline C75 & Ginkgo leaf & Morishita Jintan Co., Ltd. & 1 & $\begin{array}{l}\text { G biloba flavonoid glycoside, } \mathrm{G} \text { biloba } \\
\text { terpene latone }\end{array}$ \\
\hline C78 & Lutein & KYOWA HAKKO BIO Co., Ltd. & 1 & Lutein \\
\hline $\mathrm{C} 80$ & Peptide Meinte & FUJI OIL Co., Ltd. & 2 & Seryl tyrosine made from soybean \\
\hline C82 & The product has not been named in English yet. & Mikakuto Co., Ltd. & 2 & GABA \\
\hline $\mathrm{C} 84$ & $\begin{array}{l}\text { KAGOME tomato juice "kou-lycopene tomato } \\
\text { shiyou, syokuen-mutenka" }\end{array}$ & KAGOME Co., Ltd. & 2 & Lycopene, GABA \\
\hline C85 & Chanson Bilberry Plus & Chanson Cosmetics inc. & 1 & Bilberry extract anthocyanin \\
\hline C86 & $\begin{array}{l}\text { MAINICHI KORE 1HON EPA+DHA Fish } \\
\text { Sausage } 50\end{array}$ & Nippon Suisan Kaisha, Ltd. & 2 & DHA, EPA \\
\hline $\mathrm{C} 90$ & Health Fit Cha & Ginza stefany Inc. & 2 & $\begin{array}{l}\text { Indigestible dextrin (dietary fiber), } \\
\text { Isoflavone made from kudzu } \\
\text { (Tectorigenin) }\end{array}$ \\
\hline C91 & SAGERU & Creare Co., Ltd. & 1 & GABA \\
\hline C95 & The product has not been named in English yet. & Mikakuto Co., Ltd. & 2 & Acetic acid \\
\hline C96 & KOTHARAEX TSUBU & Fuji Sangyo Co., Ltd. & 1 & Neokotalanol \\
\hline C97 & Gussumin GABA no Chikara & Lion Corporation & 2 & GABA \\
\hline C99 & $\begin{array}{c}\text { Premier Rich Perfect Asta Hyaluronic acid } \\
\text { Powder }\end{array}$ & Asahi Group Foods, Ltd. & 1 & Hyaluronic acid $\mathrm{Na}$ \\
\hline C103 & SUNKINOU Ceramide & Sunsho Pharmaceutical Co., Ltd. & 1 & Glucosylceramide made from pineapple \\
\hline C105 & EYE GUARD & $\begin{array}{l}\text { NATURALLY HEALTH FOODS Co., } \\
\text { Ltd. }\end{array}$ & 1 & Lutein, Zeaxanthin \\
\hline C109 & Organic Enseki Kale & Enseki Aojiru Co., Ltd. & 2 & GABA $(\gamma$-Aminobutyric acid $)$ \\
\hline $\mathrm{C} 110$ & Pep-Gyu & YOSHINOYA Co., Ltd. & 2 & $\begin{array}{l}\text { Valine-Valine-Tyrosine-Proline made } \\
\text { from globin }\end{array}$ \\
\hline C111 & Daizu peptide Genen shoyu (dashi-iri) & Kikkoman Food Products Company & 2 & Soybean peptide \\
\hline
\end{tabular}


Table 2. Cont

\begin{tabular}{|c|c|c|c|c|}
\hline No. * & Product Name & $\begin{array}{l}\text { Food Business } \\
\text { Operator }\end{array}$ & $\begin{array}{c}\text { Classification of Food } \\
1 \text { Supplement } \\
2 \text { Processed Food } \\
3 \text { Fresh Produce }\end{array}$ & Functional Substance \\
\hline $\mathrm{C} 112$ & Clear Lutein & Yazuya Co., Ltd. & 1 & Lutein \\
\hline $\mathrm{C} 114$ & Latwell & ASAHI CALPIS WELLNESS Co., Ltd. & 1 & $\begin{array}{c}\text { Lactotripeptides } \\
\text { (Valine-Proline-Proline/Isoleucine-Proline-Pr }\end{array}$ \\
\hline $\mathrm{C} 120$ & Suupu you ito kanten & Ina Food Industry Co., Ltd. & 2 & Galactan made from agar (fiber) \\
\hline $\mathrm{C} 121$ & Ginkgo biloba leaf Extract Tablet & AFC Co., Ltd. & 1 & $\begin{array}{l}\text { G biloba flavonoid glycoside, G biloba } \\
\text { terpene latone }\end{array}$ \\
\hline $\mathrm{C} 123$ & Hiaruronsan C-jelly & Earth Corporation & 2 & Hyaluronic acid $\mathrm{Na}$ \\
\hline C124 & Beau Clair & $\begin{array}{l}\text { HEALTH RESEARCH } \\
\text { FOUNDATION }\end{array}$ & 1 & Lutein, Zeaxanthin \\
\hline $\mathrm{C} 125$ & Vegetable lactobacillus TAKUMINO-KIMCHI & Tokai Pickling Co., Ltd. & 2 & L. plantarum TK61406 \\
\hline $\mathrm{C} 126$ & $\begin{array}{l}\text { HAKKOU TSUBAKICHA containing } \\
\text { indigestible dextrin }\end{array}$ & YAMACHIYA Co., Ltd. & 2 & Indigestible dextrin (dietary fiber) \\
\hline $\mathrm{C} 127$ & Kiokuryokusengen & Aishitoto.Co., Ltd & 1 & $\begin{array}{c}\text { G biloba flavonoid glycoside, } \mathrm{G} \text { biloba } \\
\text { terpene latone }\end{array}$ \\
\hline $\mathrm{C} 130$ & Routeri yogurt & $\begin{array}{l}\text { OHAYO DAIRY PRODUCTS Co., } \\
\text { Ltd. }\end{array}$ & 2 & L.reuteri DSM 17938 \\
\hline $\mathrm{C} 134$ & Bifistock & EVERLIFE Co., Ltd. & 1 & Bifidobacterium lactis HN019 \\
\hline C135 & ICHOBA & MORIKAWA KENKODO Co., Ltd. & 1 & $\begin{array}{l}\text { G biloba flavonoid glycoside, } G \text { biloba } \\
\text { terpene latone }\end{array}$ \\
\hline $\mathrm{C} 138$ & arukumikata & Halmek Corporation & 1 & Undenatured type II collagen \\
\hline C161 & HMB100 & Faveurmarche Co., Ltd. & 1 & 3-Hydroxy 3-MethylButyrate (HMB) \\
\hline C162 & Everyday Dairy yogurt $<$ Low-Fat $>$ & Nippon Dairy Co-operated Co., Ltd. & 2 & Lactobacillus bifidus BB-12 (B. lactis) \\
\hline C166 & Healthy Plus Sarasara Mugicha & ITO EN, LTD. & 2 & Monoglucosyl hesperidin \\
\hline $\mathrm{C} 176$ & Suyasuya rerakkusui & Medione & 1 & $\begin{array}{l}\text { Hyperoside made from Lafua, } \\
\text { Isoqueritrin made from Lafuma }\end{array}$ \\
\hline C179 & Hitomi Management & QOL Laboratories, Inc. & 1 & Bilberry extract anthocyanin \\
\hline
\end{tabular}


Table 2. Cont.

\begin{tabular}{|c|c|c|c|c|}
\hline No. * & Product Name & $\begin{array}{l}\text { Food Business } \\
\text { Operator }\end{array}$ & $\begin{array}{c}\text { Classification of Food } \\
1 \text { Supplement } \\
2 \text { Processed Food } \\
3 \text { Fresh Produce }\end{array}$ & Functional Substance \\
\hline C180 & Lutein Hitomi no Kagayaki & $\begin{array}{c}\text { KOHKAN Pharmaceutical Institute } \\
\text { Co., Ltd. }\end{array}$ & 1 & Lutein \\
\hline C181 & Ichimokuryozen W & SOCIA Co., Ltd. & 1 & Lutein \\
\hline C182 & aojiruzanmai akatsuki & $\begin{array}{l}\text { TV SHOPPING LABORATORY Co., } \\
\text { Ltd. }\end{array}$ & 1 & L-Theanine \\
\hline C183 & The product has not been named in English yet. & Mizkan Co., Ltd. & 2 & Acetic acid \\
\hline C192 & Nyusankin shokora & LOTTE Co., Ltd. & 2 & Lactobacillus brevis NTT001 \\
\hline C197 & Hiroshima Mikan & $\begin{array}{l}\text { Hiroshima Pref. Fruit Growers } \\
\text { Cooperative Association }\end{array}$ & 3 & $\beta$-Cryptoxanthins \\
\hline C200 & Body Challenge & Ryusendo Co., Ltd. & 1 & $\begin{array}{l}\text { Ellagic acid made from African mango } \\
\text { eaves }\end{array}$ \\
\hline C201 & MUSENMAI GABA KOSHIHIKARI & ZEN-NOH PEARL RICE Co., Ltd. & 2 & $\gamma$-Aminobutyric acid (GABA) \\
\hline C205 & Suntory RURU-CHA & Suntory Beverage \& Food Limited & 2 & Inulin \\
\hline C207 & Ginkgo Biloba EX & Amway Japan G.K. & 1 & $\begin{array}{l}\text { G biloba flavonoid glycoside, G biloba } \\
\text { terpene latone }\end{array}$ \\
\hline C208 & $\begin{array}{l}\text { Mental Balance Chocolate GABA } \\
\quad<\text { Bitter }>\text { Mobile Type }\end{array}$ & EZAKI GLICO Co., Ltd. & 2 & $\gamma$-Aminobutyric acid \\
\hline C209 & Melax eye & Yawata Corporation & 1 & Lutein \\
\hline C213 & Aminomin $\mathrm{N}$ & Pharma Foods International Co., Ltd. & 1 & GABA \\
\hline C216 & Kiokuru & Suppleplus Family Co., Ltd. & 1 & $\begin{array}{l}\text { G biloba flavonoid glycoside, G biloba } \\
\text { terpene latone }\end{array}$ \\
\hline C218 & EasyTablet TERMINALIA & EC STUDIO Co., Ltd. & 1 & $\begin{array}{c}\text { Gallic acid made from Terminal nari abe } \\
\text { lyrica }\end{array}$ \\
\hline C222 & Supplement Joiner & Shiseido Company, Limited & 1 & Salmon nasal proteoglyan \\
\hline C228 & Moisture + Honey-bush Blend Tea & $\begin{array}{l}\text { SHOWA PHARMACEUTICAL Co., } \\
\text { Ltd. }\end{array}$ & 2 & N-Acetylglucosamine \\
\hline
\end{tabular}


Table 2. Cont.

\begin{tabular}{|c|c|c|c|c|}
\hline No. * & Product Name & $\begin{array}{l}\text { Food Business } \\
\text { Operator }\end{array}$ & $\begin{array}{l}\text { Classification of Food } \\
1 \text { Supplement } \\
2 \text { Processed Food } \\
3 \text { Fresh Produce }\end{array}$ & Functional Substance \\
\hline C229 & $\begin{array}{l}\text { megumi Gasseri SP Strain Yogurt Drink-type } \\
\text { Berry Mix 100g }\end{array}$ & MEGMILK SNOW BRAND Co., Ltd. & 2 & Lactobacillus gasseri SBT2055 \\
\hline $\mathrm{C} 230$ & Megami & Sun Chlorella Corp. & 1 & Astaxanthin \\
\hline C233 & Medikara supplement & Asahi Group Foods, Ltd. & 1 & Lutein \\
\hline C242 & Kenkou Benifuuki Cha & Yawata Corporation & 2 & $\begin{array}{c}\text { Methylated catechin } \\
\text { (epigallocatechin-3-O-[3-O-methyl] } \\
\text { gallate) }\end{array}$ \\
\hline C249 & shinn oishiimushimame mushisaradamame & Maruyanagi Foods Inc. & 2 & Soy isoflavone \\
\hline C251 & Glucosamine 2000 & DHC Corporation & 1 & Glucosamine hydrochloride \\
\hline C264 & Kuensan Powder & FINE JAPAN Co., Ltd. & 2 & Citric acid \\
\hline C271 & $\begin{array}{l}\text { gaba megumi rice (Special Three percent } \\
\text { milled-rice) }\end{array}$ & Tokyo foods create Co., Ltd. & 3 & GABA $(\gamma$-Aminobutyric acid $)$ \\
\hline $\mathrm{C} 272$ & $\begin{array}{l}\text { Ketsuatsu ga takame no kata no hakkou kuro } \\
\text { uuron cha }\end{array}$ & FINE JAPAN Co., Ltd. & 2 & GABA \\
\hline $\mathrm{C} 273$ & CeramiDo? & Facelabo Co., Ltd. & 1 & Glucosylceramide made from rice \\
\hline $\mathrm{C} 275$ & Q'SAI lilac-01 lactic acid bacterium & Q'SAI Co., Ltd. & 1 & Bacillus coagulans lilac- 01 \\
\hline C276 & $\begin{array}{l}\text { Todoku Tsuyosa no Nyusankin 100(Foods with } \\
\text { function claims) }\end{array}$ & ASAHI SOFT DRINKS Co., Ltd. & 2 & L. gasseri CP2305 \\
\hline C282 & Algae DHA Capsule & Nikken Sohonsha Corporation & 1 & DHA made from aurantiochytrium \\
\hline C289 & Omega A.D.E. & Suntory Wellness Limited & 1 & DHA, EPA, ARA \\
\hline C295 & Wasurerumonka & imunos Co., Ltd. & 1 & Phosphatidylserine made from soybean \\
\hline C303 & The product has not been named in English yet. & Takayuki Nishie & 2 & Acetic acid \\
\hline C318 & Mattanthermo & DHC Corporation & 1 & Monoglucosyl hesperidin \\
\hline $\mathrm{C} 321$ & Shinjyumai & Kometo Sangyo Kaisha, Ltd. & 2 & GABA \\
\hline C322 & soy isoflavone kodaizumoyashi & Meisui Bijin Factory Co., Ltd. & 3 & Soy isoflavone \\
\hline
\end{tabular}


Table 2. Cont.

\begin{tabular}{|c|c|c|c|c|}
\hline No. * & Product Name & $\begin{array}{l}\text { Food Business } \\
\text { Operator }\end{array}$ & $\begin{array}{c}\text { Classification of Food } \\
1 \text { Supplement } \\
2 \text { Processed Food } \\
3 \text { Fresh Produce }\end{array}$ & Functional Substance \\
\hline C331 & The product has not been named in English yet. & Fuji Chemical Industries Co., Ltd. & 2 & Astaxanthin \\
\hline C332 & webber naturals Lutein Plus & factorsgroup Japan LLC & 1 & Lutein \\
\hline C334 & HESPERIDIN \& COLLAGEN & EZAKI GLICO Co., Ltd. & 2 & $\begin{array}{l}\text { Monoglucosyl hesperidin, Low molecular } \\
\text { collagen peptide made from fish }\end{array}$ \\
\hline C339 & $\begin{array}{l}\text { GOMATOUNYUJITATE MINNANOMIKATA } \\
\text { DHA }\end{array}$ & Nippon Suisan Kaisha, Ltd. & 2 & EPA, DHA \\
\hline
\end{tabular}

* This number is an identification number on the CAA website. 


\subsection{Quality Assessment}

We evaluated 11 items from the AMSTAR checklist in more detail (Table 3 and Table S1 data). Interrater reliability metrics for the quality assessment indicated substantial agreement $(71.7 \%, k=0.558)$ for all 1144 items (11 items multiplied by 104 SRs).

Table 3. Quality assessment of systematic reviews (SR) of the foods with function claims.

\begin{tabular}{|c|c|c|c|c|c|c|}
\hline \multirow{2}{*}{ No. } & \multirow{2}{*}{ Items } & \multicolumn{2}{|c|}{ Before-Verification } & \multicolumn{2}{|c|}{ After-Verification } & \multirow[t]{2}{*}{$p$-Value } \\
\hline & & $N=49$ & & $N=104$ & & \\
\hline$\# 1$ & Was an 'a priori' design provided? & 2 & $4 \%$ & 89 & $86 \%$ & $<0.001$ \\
\hline$\# 2$ & $\begin{array}{l}\text { Was there duplicate study selection } \\
\text { and data extraction? }\end{array}$ & 32 & $65 \%$ & 43 & $41 \%$ & 0.006 \\
\hline$\# 3 a$ & $\begin{array}{l}\text { Was a comprehensive literature } \\
\text { search performed? }\end{array}$ & $3.8 \pm 1.8^{*}$ & $(2-15)$ & $3.8 \pm 1.8$ * & $(2-17)$ & 1.000 \\
\hline$\# 3 b$ & $\begin{array}{l}\text { Did the SR use the MESH terms and } \\
\text { related search function to detect } \\
\text { comprehensively? }\end{array}$ & 26 & $53 \%$ & 49 & $47 \%$ & 0.492 \\
\hline$\# 4$ & $\begin{array}{l}\text { Was the status of publication used as } \\
\text { an inclusion criterion? }\end{array}$ & 12 & $24 \%$ & 3 & $3 \%$ & $<0.001$ \\
\hline$\# 5$ & $\begin{array}{l}\text { A list of included and excluded } \\
\text { studies should be provided. }\end{array}$ & 49 & $100 \%$ & 102 & $98 \%$ & 0.329 \\
\hline \#6 & $\begin{array}{l}\text { Were the characteristics of the } \\
\text { included studies provided? }\end{array}$ & 41 & $84 \%$ & 91 & $88 \%$ & 0.521 \\
\hline$\# 7$ & $\begin{array}{l}\text { Was the scientific quality of the } \\
\text { included studies assessed and } \\
\text { documented? }\end{array}$ & 36 & $73 \%$ & 61 & $59 \%$ & 0.076 \\
\hline$\# 8$ & $\begin{array}{l}\text { Was the scientific quality of the } \\
\text { included studies used appropriately } \\
\text { in formulating conclusions? }\end{array}$ & 13 & $27 \%$ & 27 & $26 \%$ & 0.940 \\
\hline$\# 9$ & $\begin{array}{l}\text { Were the methods used to combine } \\
\text { the findings of studies appropriate? }\end{array}$ & $5 / 9^{* *}$ & $56 \%$ & $12 / 13^{* *}$ & $92 \%$ & 0.116 \\
\hline$\# 10$ & $\begin{array}{l}\text { Was the likelihood of publication } \\
\text { bias assessed? }\end{array}$ & 6 & $12 \%$ & 13 & $13 \%$ & 0.964 \\
\hline$\# 11$ & Was the conflict of interest stated? & 38 & $78 \%$ & 26 & $25 \%$ & $<0.001$ \\
\hline $\begin{array}{l}\text { aluation } \\
\text { score }\end{array}$ & pts./11 & $6.2 \pm 1.8$ & $(2-11)$ & $5.0 \pm 1.9$ & $(1-11)$ & $<0.001$ \\
\hline
\end{tabular}

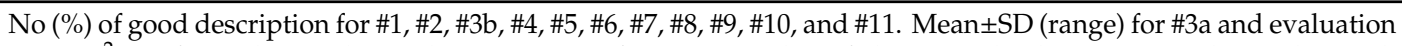
score. $\chi^{2}$ test for each item, but Fisher's exact test for \#9. ${ }^{*}$ Number of electronic databases. ${ }^{* *}$ Article number with meta-analysis is 9 in before-evaluation and 13 in after-evaluation.

Overall, there was an increase over time in evaluation score. The average of the quality score for before-SRs and after-SRs was $6.2 \pm 1.8$ and $5.0 \pm 1.9$, respectively, which was a statistically significant decrease $(p<0.001)$.

Regarding category of each item in the after-evaluation group, four items (\#1, \#5,\#6, and \#9) were a good description $(80 \%-100 \%)$, one item (\#7) was a poor description $(50 \%-79 \%)$, and six items (\#2, $\# 3 \mathrm{~b}, \# 4, \# 8, \# 10$, and $\# 11$ ) were a very poor description ( $0 \%-49 \%)$.

There was a good description and/or implementation for the following items: "Was an 'a priori' design provided?" (before-, $4 \%$ and after-, $86 \%, p<0.001$ ); "A list of included and excluded studies should be provided." (before-, 100\% and after-, 98\%, $p=0.329$ ); "Were the methods used to combine the findings of studies appropriate?" (before-, $56 \%$ and after, $92 \%, p=0.116$ ); and "Were the characteristics 
of the included studies provided?" (before-, $84 \%$ and after-, $88 \%, p=0.521$ ). These items were still a good description or improving in the after-evaluation.

There continued to be a poor description and/or implementation for the item, "Was the scientific quality of the included studies assessed and documented?" (before-, 73\% and after-, 59\%, $p=0.076$ ).

The other items were a very poor description and/or implementation: "Was there duplicate study selection and data extraction?" (decreased from $65 \%$ to $41 \%, p<0.01$ ); "Did the SR use the MESH terms and related search function to detect comprehensively?" (decreased from $53 \%$ to $47 \%, p=0.492$ ); "Was the status of publication used as an inclusion criterion?" (decreased from $24 \%$ to $3 \%, p<0.001$ ); "Was the scientific quality of the included studies used appropriately in formulating conclusions?" (decreased from $27 \%$ to $26 \%, p=0.94$ ); "Was the likelihood of publication bias assessed?" (increased from $12 \%$ to $13 \%, p=0.964$ ); and "Was the COI stated?" (all decreased; from $78 \%$ to $25 \%, p<0.001$ ).

According to one component of \#3a, "which databases did the SR use or number of the other databases?", the number of used databases was the same between before- and after-evaluation (mean $\pm \mathrm{SD}$ was $3.8 \pm 1.8, p=1.000$ ). According to respective before- and after-evaluations, the high utility databases were PubMed (93.9\% and 100\%), JDream III (in Japanese databases, 79.6\% and 60.6\%), Ichushi-Web (in Japanese databases, $67.3 \%$ and $64.4 \%$ ), The Cochrane Library (with CENTRAL, $49.0 \%$ and $55.8 \%$ ), and UMIN-CTR (Japanese clinical trial registry, $18.4 \%$ and $23.1 \%$ ).

\section{Discussion}

This is the first prospective before- and after- SR of SRs of the FFC registered on the CAA website in Japan.

The FFC in Japan is an original and unique system regarding health claims. A food business operator must submit a completed notification and related documents to the Secretary-General of the CAA 60 days prior to the launch date. Therefore, all consumers can check all content such as safety, functional mechanism, and effectiveness (i.e., total evidence) of the product, resulting in high transparency.

We propose that this study will be helpful to researchers and government officials who want to know about new health claims in advanced countries. We expected that the total quality of after-SRs might be improved significantly by the CAA's verification report in 2016 [10], but this study instead showed deterioration in quality. Therefore, it is necessary to discuss the interpretation of these findings and propose a practical future strategy for this issue.

\subsection{Quality Assessment of Target SRs}

Overall, the quality of articles significantly decreased in conduct and reporting. Although four items (\#1, \#5, \#6, and \#9) in the after-SRs group were improving or remained a good description, another seven items were poor or very poor.

The methodology for most SRs did not attempt to include so-called grey literature by the use of many other types of databases and classical literature searches. Grey literature was defined here as studies that are unpublished, have limited distribution, and/or are not included in the bibliographical retrieval system [16]. The importance of including grey literature in all SRs has been previously discussed [17]. Implementers of SRs need to recognize the importance of also searching grey literature.

"Was the likelihood of publication bias assessed?" also remained flawed in the assessment process. Additionally, many SRs used "at least two electronic sources", but these were only Japanese databases and/or not the more traditional English databases like EMBASE or MEDLINE. Furthermore, it has been pointed out that there is a bias in coverage with only one database (i.e., PubMed) [18,19]. Researchers performing SRs therefore need to use multiple databases.

Publication bias remains an area of contention amongst researchers who assess the quality of SRs $[20,21]$. However, it remains a research priority because it is unclear what impact publication bias has on making decisions in healthcare [9]. We assume that the new FFC guideline provides a better description of how to assess publication bias, especially for a qualitative SR. 
"Was the conflict of interest stated?" was a serious problem. Although most SRs described a part of the COI, they did not include all necessary information such as the SR's sponsor, SR's funding, author's affiliation, SR's outsourcing information (research agency), supervision allowance, and consulting fees for an SR. In fact, the targeted SRs included those that were conducted only by the company itself, those conducted by other companies, such as raw material makers, those conducted by a research agency, and those supervised by academia researchers. We assume that the primary reason reviewers of quality assessment judged many SRs as 'unclear or inadequately described' (Can't answer) was because they could not cover these elements properly. The International Committee of Medical Journal Editors (ICMJE) emphasizes that when authors submit a manuscript of any type or format, they are responsible for disclosing all financial and personal relationships that might bias or be seen to bias their work [22].

"Was there duplicate study selection and data extraction?" was also a very poor description. This was described clearly in the CAA's verification report in 2016 [10]. Because everyone makes mistakes occasionally, there should be at least two independent data extractors, and a consensus procedure for disagreements should be in place. It was not clear why two additional researchers did not perform independent assessments for some of the SRs.

"Did the SR use the MESH terms and related search function to detect comprehensively?" got worse in the after-evaluation. The guideline [4] instructs that "To search comprehensively, a search formula made by combining free items and controlled terms (including MeSH for PubMed) appropriately will be set per bibliographic databases." In addition, the report [10] points out that it is essential to design an optional search formula by combining keywords and thesauruses (such as MeSH) appropriately for each clinical question according to each database characteristic.

We assume that there were multiple reasons for the quality declining significantly. The FFC system is just a notification, so the CAA does not evaluate the safety and effectiveness of a submitted product. The number of notifications has increased since the system was launched in 2015 to lead sales promotions, but the reason for this might be that multiple companies had purchased copies of the SRs that had already been accepted by the CAA and submitted them to the CAA as basis material for the evidence. Therefore, many low-level SRs may have been contained in the FFC system, so the quality of after-evaluation might have deteriorated.

\subsection{Validity and Reliability of Quality Assessment by AMSTAR Checklist}

For the before-evaluation, we adopted a measurement tool used for the 'assessment of multiple SR' (AMSTAR checklist). The "R-AMSTAR" [23] was also developed as an approach to minimize bias of any kind in SRs. In terms of interrater reliability and validity, the AMSTAR score was very high compared with scores from other tools. In terms of feasibility, it was very appropriate that scoring time of the AMSTAR was short (between 10 and $20 \mathrm{~min}$ ) [24]. Furthermore, a recent methodological study showed that AMSTAR and the risk of bias in systematic reviews (ROBIS) had similar interrater reliability but differed in their construct and applicability [25].

Although we had one consensus-training session and all reviewers had conducted a quality assessment of SRs more than once, the interrater reliability metrics for the quality assessment indicated substantial agreement was average $71.7 \%, k=0.558$. It can also be interpreted that there were many SRs for which the quality reviewers were confused as to whether it was a "Yes", "No", or "Can't answer".

Additionally, the reviewers seemed to have some ambiguity about the details of each item. Recently, a quality assessment check list, AMSTAR 2, was developed that allows for individual responses that do not impart judgment for each item [13]. AMSTAR 2 retains 10 of the original domains, has 16 items in total (compared with 11 in the original), has simpler response categories than the original AMSTAR, includes a more comprehensive user guide, and includes the identification of high-quality SRs. It might be useful to evaluate the detail quality for each item of the SRs in a future study. 


\subsection{Future Research Challenge to Improve the Quality of SRs of the FFC}

Table 4 shows the future research challenge for studies on the health enhancement effects of the FFC and related healthy foods. We assumed that there are three important dimensions and six tasks due to improved systematic reviews. Regarding the food industry, researchers must study the current standard rule of an SR (i.e., AMSTAR 2, PRISMA checklist, and PRISMA-NMA checklist for meta-analysis) [26] before research is conducted. If an applicant is concerned about the implementation of an SR, they should immediately consult with experts on research methodology, which will avoid creating inappropriate SRs. Moreover, since the CAA only performs formal confirmation of documents, the methodology of the SRs that had already been notified was not always correct. Therefore, if another company's SR is reused for a notification, it becomes necessary for an applicant to carefully examine the SR before deciding to confidently introduce its own product to the market.

Table 4. Research challenge on systematic review of the foods with function claims.

\begin{tabular}{|c|c|}
\hline \multicolumn{2}{|c|}{ For food industry } \\
\hline$\# 1$ & The applicants should conduct research based on AMSTAR 2 checklist. \\
\hline$\# 2$ & The applicants should conduct research based on PRISMA checklist and PRISMA-NA (for meta-analysis). \\
\hline$\# 3$ & $\begin{array}{l}\text { The applicants should examine its quality when using the SR of another company that had already been } \\
\text { accepted by the CAA. }\end{array}$ \\
\hline$\# 4$ & The applicants should consult with academia researchers for unclear points in methodology. \\
\hline \multicolumn{2}{|c|}{ For academia } \\
\hline \#5 & $\begin{array}{l}\text { Academic researchers should provide support for food companies and others to implement the } \\
\text { SR properly. }\end{array}$ \\
\hline$\square$ & Study plan (study selection and data extraction, search strategy, and evaluation method of bias risk) \\
\hline$\square$ & $\begin{array}{l}\text { Implementation (assessment of publication bias, and formulating conclusion based on methodological } \\
\text { rigor quality) }\end{array}$ \\
\hline$\square$ & Description (conflict of interest) \\
\hline \multicolumn{2}{|c|}{ For the Consumer Affairs Agency in Japan } \\
\hline \#6 & $\begin{array}{l}\text { The authorities should evaluate not only the formal confirmation* in the document but also the quality } \\
\text { (certain level or higher) of the SR. }\end{array}$ \\
\hline
\end{tabular}

* Currently, the government intends to deregulate in food industy, so the CAA cannot examine the quality of each SR.

Academia should provide its own support for food companies and other companies to implement the SR properly, and academic researchers will need to continue to convey appropriate SR methodologies to the food industry. In the present study, it became clear that there were many methodological deficiencies in targeted SRs. The FFC system in Japan relies on one SR or one clinical trial, such as a $\mathrm{RCT}$ as a basis for efficacy. However, a Japanese research group recently identified problems with the reporting quality and associated issues for RCTs of the FFC [27]. There was insufficient information on items associated with sample size, allocation and blinding, results of outcomes and estimation, generalizability of the results, and study registration numbers. Because it is a notification system, it is essential for academic researchers, including our group, to monitor all SRs and clinical trials for the FFC.

Considering that the Japanese government has introduced the world's most advanced FFC system as part of its growth strategy (i.e., deregulation), it may be difficult for the CAA to review individual SRs. Therefore, to protect consumers, we assume it is necessary to confirm that the notification SR is above a certain level of quality.

Either way, even for an SR that has already been accepted, it will be necessary to issue the latest (updated) version 5 to 10 years later. The prospect of this future requirement will encourage all existing SRs to be conducted by scientifically correct methodologies. 


\section{Limitations}

This review had several limitations that should be acknowledged. First, publication bias was possible because there was not enough use of multiple databases for each SR. Second, we could not perform an evaluation using the PRISMA checklist. Third, our study design focused on the quality of SRs; therefore, we could not validly assess the safety or the functional mechanism of any of the products reviewed in the SRs. Lastly, because we did not conduct a retrospective analysis of the quality of "primary studies cited or used as references" that were described in submitted SRs, the effectiveness of functional substances or finished products could not be addressed.

\section{Conclusions}

Overall, the quality of methodology and reporting in after-SRs based on the FFC was poorer than that based on before-SRs. In particular, there were very poor descriptions and/or implementation of study selection, data extraction, search strategy, evaluation methodology for risk of bias, assessment of publication bias, and formulating conclusions based on methodological rigor and scientific quality of the included studies.

To develop SRs of the FFC and launch a similar global food claim notification system, the following factors will be important: (i) applicants will need to use some global standard checklist such as AMSTAR 2, PRISMA, or PRISMA-NMA; (ii) applicants will need to critically examine the quality when using another applicant's SR; (iii) academic researchers should support the food industry in order to perform an SR and/or clinical trial properly; and (iv) country authorities should confirm that the notification SR is above a certain level of quality.

Supplementary Materials: The following are available online at http://www.mdpi.com/2072-6643/11/7/1583/s1, Table S1: Results of quality assessment of all targeted systematic reviews.

Author Contributions: H.K. conceived the study and takes responsibility for the quality assessment. H.K. made a brief summary of included studies and data extraction. K.T., H.O., and H.T.-O. were the guarantors. H.K. designed the study and performed the searches. T.Y., M.S., and J.K. acquired the data. H.K. described the brief summary from each article. Y.W., T.Y., M.S., J.K., and H.K. assessed the quality of articles. All authors critically revised the manuscript for important intellectual content.

Funding: This study was supported by a Grant-in-Aid for Scientific Research (C) Number 17K00885 from the Ministry of Education, Culture, Sports, Science and Technology (MEXT), Japan, 2017-2018.

Acknowledgments: We would like to express our appreciation to Michiko Ishida and Tatsunosuke Gomi (paperwork), and Mari Makishi and Satoko Sayama (searching advice) for their assistance with this study.

Conflicts of Interest: The datasets used and/or analyzed during the current study are available from the corresponding author upon reasonable request. When there was a case of conflict of interest (COI) (e.g., consulting on the study, shareholder of the firm), the reviewer did not evaluate the study directly, and other reviewers performed the evaluation. HK supervised SRs on five corporations (FANCL CORPORATION, Morishita Jintan Co., Ltd., KAGOME Co., Ltd., Fujifilm Corporation, and AJINOMOTO AGF, Inc.) and was compensated for that work. YT analyzed an SR on three corporations (KAGOME Co., Ltd., Fujifilm Corporation, and AJINOMOTO AGF, Inc.) and was compensated for that work. Therefore, HK and YT did not evaluate SRs involving those corporations, and instead other authors did the work.

$\begin{array}{ll}\text { Abbreviations } \\ \text { SR } & \text { Systematic review } \\ \text { CAC } & \text { Codex Alimentarius Commission } \\ \text { FAO } & \text { Food and Agriculture Organization } \\ \text { WHO } & \text { World Health Organization } \\ \text { FFC } & \text { Foods with Function Claims } \\ \text { CAA } & \text { Consumer Affairs Agency } \\ \text { FOSHU } & \text { Foods for Specified Health Uses } \\ \text { FNFC } & \text { Foods with Nutrient Function Claims } \\ \text { RCT } & \text { Randomized controlled trial } \\ \text { MeSH } & \text { Medical subject headings }\end{array}$




\section{References}

1. The CODEX Alimentarius Committee. About Codex Alimentarius. 2018. Available online: http://www.fao. org/fao-who-codexalimentarius/about-codex/en/ (accessed on 6 July 2018).

2. The CODEX Alimentarius Committee. Guidelines for Use of Nutrition and Health Claims. 1997. Available online: http://www.fao.org/fao-who-codexalimentarius/sh-proxy/en/?lnk=1\&url=https\%253A\%252F\%252 Fworkspace.fao.org\%252Fsites\%252Fcodex\%252FStandards\%252FCAC\%2BGL\%2B23-1997\%252FCXG_02 3e.pdf (accessed on 6 July 2018).

3. Consumer Affairs Agency. Government of Japan. Introduction. 2015. Available online: http://www.caa. go.jp/policies/policy/food_labeling/about_foods_with_function_claims/pdf/150810_1.pdf (accessed on 10 August 2018).

4. Consumer Affairs Agency. Government of Japan. Guideline. 2015. Available online: http://www.caa.go.jp/polici es/policy/food_labeling/foods_with_function_claims/pdf/food_with_function_clains_180328_0001.pdf (accessed on 20 August 2018).

5. National Information Center on Health Services Research and Health Care Technology. Systematic Reviews Definition. 2017. Available online: http://www.nlm.nih.gov/nichsr/hta101/ta101014.html (accessed on 15 September 2018).

6. Page, M.J.; Shamseer, L.; Altman, D.G.; Tetzlaff, J.; Sampson, M.; Tricco, A.C.; Catalá-López, F.; Li, L.; Reid, E.K.; Sarkis-Onofre, R.; et al. Epidemiology and Reporting Characteristics of Systematic Reviews of Biomedical Research: A Cross-Sectional Study. PLoS Med. 2016, 13, e1002028. [CrossRef] [PubMed]

7. Shamser, L.; Moher, D.; Clarke, M.; Ghersi, D.; Liberati, A.; Petticrew, M.; Shekelle, P.; Stewart, L.A. Preferred reporting items for systematic review and meta-analysis protocol (PRISMA-P) 2015: Elaboration and explanation. BMJ 2015, 349, g7647. [CrossRef] [PubMed]

8. Kamioka, H.; Tsutani, K.; Hideki, O.; Yoshizaki, T.; Kitayuguchi, J.; Shimada, M.; Tang, W.; Takano-Ohmuro, H. Quality of systematic reviews of the Foods with Function Claims registered at the Consumer Affairs Agency Web site in Japan: A prospective systematic review. Nutr. Res. 2017, 40, 21-31. [CrossRef] [PubMed]

9. Shea, B.J.; Grimshaw, J.M.; A Wells, G.; Boers, M.; Andersson, N.; Hamel, C.; Porter, A.C.; Tugwell, P.; Moher, D.; Bouter, L.M. Development of AMSTAR: A measurement tool to assess the methodological quality of systematic reviews. BMC Med. Res. Methodol. 2007, 7, 10. [CrossRef] [PubMed]

10. Consumer Affairs Agency, Government of Japan. Verification of Scientific Evidence on Effectiveness of the System of "Foods with Function Claim": Assessment of the Submitted Systematic Literature Reviews (Digest Edition). 2016. Available online: http://www.caa.go.jp/policies/policy/food_labeling/about_foods_with_fun ction_claims/pdf/food_with_function_report_0003.pdf (accessed on 1 July 2018).

11. Liberati, A.; Altman, D.G.; Tetzlaff, J.; Mulrow, C.; Gotzsche, P.C.; Ioannidis, J.P.; Clarke, M.; Devereaux, P.J.; Kleijnen, J.; Moher, D. The PRISMA statement for reporting systematic reviews and meta-analyses of studies that evaluate health care interventions: Explanation and elaboration. Ann. Intern. Med. 2009, 151, W65-W94. [CrossRef] [PubMed]

12. Moher, D.; Jones, A.; Lepage, L. Use of the CONSORT statement and quality of reports of randomized trials: A comparative before-and-after evaluation. JAMA 2001, 285, 1992-1995. [CrossRef] [PubMed]

13. Shea, B.J.; Reeves, B.C.; Wells, G.; Thuku, M.; Hamel, C.; Moran, J.; Moher, D.; Tugwell, P.; Welch, V.; Kristjansson, E.; et al. AMSTAR 2: A critical appraisal tool for systematic reviews that include randomised or non-randomised studies of healthcare interventions, or both. BMJ 2017, 358, j4008. [CrossRef] [PubMed]

14. Hopewell, S.; Ravaud, P.; Baron, G.; Boutron, I. Effect of editors' implementation of CONSORT guidelines on the reporting of abstracts in high impact medical journals: Interrupted time series analysis. BMJ 2012, 344, e4178. [CrossRef] [PubMed]

15. International Prospective Register of Systematic Reviews. Protocol. 2017. Available online: http://www.crd. york.ac.uk/prospero/ (accessed on 21 December 2017).

16. Last, J.M. A Dictionary of Epidemiology, 4th ed.; Oxford University Press: New York, NY, USA, 2001.

17. McAuley, L.; Pham, B.; Tugwell, P.; Moher, D. Does the inclusion of grey literature influence the estimates of intervention effectiveness reported in meta-analyses? Lancet 2000, 356, 1228-1231. [CrossRef]

18. Loria, A.; Arroyo, P. Language and country preponderance trends in MEDLINE and its causes. J. Med. Libr. Assoc. 2005, 93, 381-385. [PubMed] 
19. Xu, Q.; Boggio, A. Countries' biomedical publications and attraction scores: A PubMed-based assessment. F1000Research 2015, 3, 292. [CrossRef] [PubMed]

20. Müller, K.F.; Briel, M.; D’ Amario, A.; Kleijnen, J.; Marusic, A.; Wager, E.; Antes, G.; Von Elm, E.; Lang, B.; Motschall, E.; et al. Defining publication bias: Protocol for a systematic review of highly cited articles and proposal for a new framework. Syst. Rev. 2013, 2, 34. [CrossRef] [PubMed]

21. Chan, A.W.; Tetzlaff, J.M.; Altman, D.G.; Laupacis, A.; Gøtzsche, P.C.; Krleža-Jerić, K.; Hróbjartsson, A.; Mann, H.; Dickersin, K.; Berlin, J.A.; et al. SPIRIT 2013 Statement: Defining standard protocol items for clinical trials. Ann. Intern. Med. 2013, 158, 200-207. [CrossRef] [PubMed]

22. International Committee of Medical Journal Editors. Recommendations for the Conduct, Reporting, Editing, and Publication of Scholarly Work in Medical Journals: Updated December 2017. Available online: http://www.icmje.org/icmje-recommendations.pdf (accessed on 6 November 2018).

23. Kung, J.; Chiappelli, F.; Cajulis, O.O.; Avezova, R.; Kossan, G.; Chew, L.; Maida, C.A. From systematic reviews to clinical recommendations for evidence-based health care: Validation of revised assessment of multiple systematic reviews (R-AMSTAR) for grading of clinical relevance. Open Dent. J. 2010, 4, 84-91. [CrossRef] [PubMed]

24. Pieper, D.; Buechter, R.B.; Li, L.; Prediger, B.; Eikermann, M. Systematic review found AMSTAR, but not R (revised)-AMSTAR, to have good measurement properties. J. Clin. Epidemiol. 2015, 68, 574-583. [CrossRef] [PubMed]

25. Banzi, R.; Cinquini, M.; Gonzalez-Lorenzo, G.; Pecoraro, V.; Capobussi, M.; Minozzi, S. Quality assessment versus risk of bias in systematic reviews: AMSTAR and ROBIS had similar reliability but differed in their construct and applicable. J. Clin. Epidemiol. 2018, 99, 24-32. [CrossRef] [PubMed]

26. Hutton, B.; Salanti, G.; Caldwell, D.M.; Chaimani, A.; Schmid, C.H.; Cameron, C.; Ioannidis, J.P.; Straus, S.; Thorlund, K.; Jansen, J.P.; et al. The PRISMA extension statement for reporting of systematic reviews incorporating network meta-analyses of health care interventions: Checklist and explanations. Ann. Intern. Med. 2015, 162, 777-784. [CrossRef] [PubMed]

27. Tanemura, N.; Hamadate, N.; Urushibara, H. Evaluation of randomized controlled trials of foods with functional claims request: The learning outcomes from studies in Japan. J. Funct. Foods 2018, 42, 248-253. [CrossRef]

(C) 2019 by the authors. Licensee MDPI, Basel, Switzerland. This article is an open access article distributed under the terms and conditions of the Creative Commons Attribution (CC BY) license (http://creativecommons.org/licenses/by/4.0/). 